\title{
Supplemental Information of
}

\section{The multiple-valence and visible to near-infrared photoluminescence of Manganese in $\mathrm{ZnGa}_{2} \mathrm{O}_{4}$ :}

A first principles study

Qiaoling Chen, ${ }^{\dagger,+} \boldsymbol{\Phi}$ Longbing Shang, ${ }^{\dagger,+,}$ Haoming $\mathrm{Xu}^{\dagger,+, \uparrow}$ Chonggeng Ma, ${ }^{\S}$ and Chang-Kui Duan ${ }^{*, \dagger, \uparrow, \uparrow}$

$\dagger$ Hefei National Laboratory for Physical Sciences at the Microscale and School of Physical Sciences, University of Science and Technology of China, Hefei 230026, China $\ddagger C A S$ Key Laboratory of Microscale Magnetic Resonance, University of Science and Technology of China, Hefei 230026, China

\CAS Center for Excellence in Quantum Information and Quantum Physics, University of Science and Technology of China, Hefei 230026, China $\S$ School of Optoelectronic Engineering 83 CQUPT-BUL Innovation Institute, Chongqing University of Posts and Telecommunications, Chongqing 400065, China

E-mail: ckduan@ustc.edu.cn 


\section{Note 1 Charge corrections}

Accurate defect formation energies are vital to understand the properties of materials. Charged defects introduces finite-size artifacts in the framework of periodic density functional theory. Here, we follow the method proposed by Durrant et. al., where the the Lany-Zunger electrostatic image interaction combined with a potential alignment between neutral systems $\Delta V_{\mathrm{AB}}$ (the original bulk supercell, i.e. the A configuration and the unrelaxed supercell containing the defect, i.e. the B configuration) can provide reliable corrections. ${ }^{1}$ The Lany-Zunger correction ${ }^{2,3}$ for electrostatic image interaction is given as

$$
\begin{gathered}
E_{\mathrm{corr}}^{\mathrm{LZ}}=f^{\mathrm{LZ}} \frac{q^{2} \alpha_{M}}{2 \epsilon L} \\
f^{\mathrm{LZ}}=1-c_{\mathrm{sh}}\left(1-\epsilon^{-1}\right)
\end{gathered}
$$

where $f^{\mathrm{LZ}}$ is the corrections by making a practical approximation to quadrupole moment and the shape factor, $c_{\mathrm{sh}}$ depends on the shape of the defect containing the supercell. ${ }^{3}$ For the cubic supercells, the value of $c_{\mathrm{sh}}$ is close to $1 / 3 . \alpha_{M}$ is the Madelung constant, $\epsilon$ is the dielectric constant and $L=\Omega^{1 / 3}$ is the linear supercell dimension. Since the ionic relaxation provides additional dielectric screening of the interactions between the replicas, the static dielectric constant $\epsilon_{0}$ is applied for defect formation energies.

For the potential alingment, the removal or addition of atoms from the supercell alone $\Delta V_{\mathrm{AB}}$ is the dominant remaining finite-size error once the image interaction correction has been applied. Besides, $\Delta V_{\mathrm{AB}}$ can be readily approximated simply by placing an atom of the type being exchanged in a periodic supercell of the same size. The native defects levels of $\mathrm{Ga}_{\mathrm{Zn}}$ and $V_{\mathrm{Zn}}$ are close with CBM and VBM, respectively and the electrons and holes cannot

be totally shield, which leads to the long ranged Coulomb interaction effects on electronic 
potential. Thus, we take the atomic approximation for consistency and the potential energies of defects are plotted in Fig. S1 by placing the corresponding atom in an empty periodic supercell. The detailed corrections to total energy are listed in Tab. S1.

\section{Note 2 Tanabe-Sugano model}

Traditionally, the optical properties of transition metal ions strongly depend on the ligand field and the Tanabe-Sugano diagrams based on ligand field theory for transition metal surrounded by ligands of approximately $O_{h}$ or $T_{d}$ symmetry have been successfully applied to the qualitative analysis of luminescent properties of transition metal ions in crystal. Usually, the $t_{2}$ and $e$ orbitals have been assumed to have nearly pure $d$-character, ${ }^{4}$ and then three empirical parameters, the Racah parameters $B$ and $C$ and the ligand field strength $\Delta$, are required to completely describe the multielectron term energy levels. Generally, these parameters are determined by fitting to experimentally measured optical spectra, and the results strongly depend on the availability of high-quality spectra and correct identification of bands.

First principles calculations have emerged as a predictive tool in the identification and characterization of defects. The constrained occupancy approach and spin multiplicity control combined with $\triangle \mathrm{SCF}$ method have been succesfully applied to varify and further predict the low excitations and emissions, such as excitons, $\mathrm{Cr}^{3+}, \mathrm{Bi}^{3+}, \mathrm{Ce}^{3+}$ and charge transfer transition of $\mathrm{Eu}^{2+}-\mathrm{Tb}^{3+}{ }^{5-8}$ However, in the DFT single electionic framework, those excited states dominated by multi-slater determinant wavefunctions cannot be obtained for luminescent varification. Thus, in our work, the DFT combined with Tanabe-Sugano diagrams is applied for detailed spectrum of transition metal activators, as shown in Fig. S4. The Racah parameter ratios $C / B=4.48,4.60,4.50$ (http://wwwchem.uwimona.edu. jm/courses/Tanabe-Sugano/TSspread.html) are adopted to produce Tanabe-Sugano diagrams for $\mathrm{Mn}^{2+, 3+, 4+}$, respectively. Those values are similar to varies values appeared in 
the literatures.

For $\mathrm{Mn}^{2+}$ substituted at tetrahedral $\mathrm{Zn}^{2+}$ sites, by majority-spin $e^{2} t_{2}^{3}$ occupation, the ligand filed strength $\Delta$ can be obtained as the difference of average $t_{2}$ orbital energies and average $e$ orbital energies. Thus, the ligand field strength $\Delta$ is calculated to be $0.82 \mathrm{eV}$ at HSE06 level in the tetrahedral ligands. Our first principles calculation can determine a line in Tanabe-Sugano diagram for each energy level and the lines of ${ }^{4} \mathrm{~T}_{1}$ and ${ }^{2} \mathrm{~T}_{2}$ are determined by

$$
\begin{aligned}
& k=E\left({ }^{4} \mathrm{~T}_{1}\right) / \Delta, \quad E\left({ }^{4} \mathrm{~T}_{1}\right) / B=k \cdot \Delta / B \\
& k=E\left({ }^{2} \mathrm{~T}_{2}\right) / \Delta, \quad E\left({ }^{2} \mathrm{~T}_{2}\right) / B=k \cdot \Delta / B
\end{aligned}
$$

where the vertical coordinates is $E / B$ and the horizontal coordinates is $\Delta / B$ in TanabeSugano diagram. Then, the corresponding intersection points are labeled in Fig. S4(a) and the two $x$-values are close to each other and set a rather narrow range $\Delta / B=9.4 \sim 9.6$. Thus, the electronic repulsion parameter can be obtained as $B=689 \sim 704 \mathrm{~cm}^{-1}$ at HSE06 level.

For $\mathrm{Mn}^{3+}$ substituted at octahedral $\mathrm{Ga}^{3+}$ sites, the ligand field strength $\Delta=2.30 \mathrm{eV}$ at HSE06 level is obtained as the difference of average $t_{2}$ orbital energies and average $e$ orbital energies in the octahedral ligands. The extra $0.5 e$ is added to shift all five $3 d$ orbitals into the bandgap after obtaining the required geometric structure. Besides, the energy level of ${ }^{5} \mathrm{~T}_{2}$ relative to ground state energy $E\left({ }^{5} \mathrm{~T}_{2}\right)-E\left({ }^{5} \mathrm{E}\right)=2.28 \mathrm{eV}$ is close to the ligand field strength, which is coincident with the previous derivation under the approximation of $O_{h}$ or $T_{d}$ site symmetry and $t_{2}$ and $e$ wavefunctions having pure $d$-character. ${ }^{4}$ Similarly, the DFT calculation can determine the lines of ${ }^{3} \mathrm{~T}_{1}$ and ${ }^{1} \mathrm{~T}_{2}$ as

$$
k=E\left({ }^{3} \mathrm{~T}_{1}\right) / \Delta, \quad E\left({ }^{3} \mathrm{~T}_{1}\right) / B=k \cdot \Delta / B
$$




$$
k=E\left({ }^{1} \mathrm{~T}_{2}\right) / \Delta, \quad E\left({ }^{1} \mathrm{~T}_{2}\right) / B=k \cdot \Delta / B
$$

The corresponding intersections of ${ }^{3} \mathrm{~T}_{1}$ and ${ }^{1} \mathrm{~T}_{2}$ levels in Fig. S4(b) are obtained to determine the range of $\Delta / B=21.4 \sim 22.0$ and the electronic repulsion $B=843 \sim 867 \mathrm{~cm}^{-1}$ at HSE06 level. The same procedures are performed for $\mathrm{Mn}^{4+}$ substituted at octahedral $\mathrm{Ga}^{3+}$ sites. However, the five $3 d$ orbitals cannot simutaneously in the bangap and the ligand field strength is estimated by $\Delta=E\left({ }^{4} \mathrm{~T}_{2}\right)-E\left({ }^{4} \mathrm{~A}_{2}\right)=2.77 \mathrm{eV},{ }^{4}$ then $\Delta / B=28.2$ and the electronic repulsion $B=792 \mathrm{~cm}^{-1}$ at HSE06 level. Detailed values are listed in Table S2.

Finally, we briefly discuss the relationship between ligand field strength and average Mn$\mathrm{O}$ bond length of $\mathrm{Mn}^{2+}, \mathrm{Mn}^{3+}$ and $\mathrm{Mn}^{4+}$ valence states. For tetrahedral Mn substituted at $\mathrm{Zn}^{2+}$ site, $\mathrm{Mn}^{2+}$ and $\mathrm{Mn}^{3+}$ states can exist with Fermi energy. In the ground state geometric configuration of $\mathrm{Mn}^{2+}$ in tetrahedral ligands, the four $\mathrm{Mn}-\mathrm{O}$ bond lengths are $2.02 \AA$. The ligand field strength $\Delta$ is $0.75 \mathrm{eV}$ at GGA $+U$ level and $0.82 \mathrm{eV}$ at HSE06 level. The four $\mathrm{Mn}-\mathrm{O}$ bond lengths are $1.93 \AA$ in the ground state geometric configuration of $\mathrm{Mn}^{3+}$ in tetrahedral ligands and the ligand field strength $\Delta$ is $0.93 \mathrm{eV}$ at GGA $+U$ level and $0.98 \mathrm{eV}$ at HSE06 level. The extra $1 e$ is added to ensure all five $3 d$ orbitals in the bandgap. The ligand field strength is converted by $\Delta_{\mathrm{Oh}}=(9 / 4) \Delta_{\mathrm{Td}}{ }^{9}$ to compare with those for Mn at octahedral site. For octahedral Mn substituted at $\mathrm{Ga}^{3+}$ site, $\mathrm{Mn}^{2+}, \mathrm{Mn}^{3+}$ and $\mathrm{Mn}^{4+}$ states can exist with Fermi energy. The six Mn-O bond lengths are all $2.14 \AA$ for the ground state geometric configuration of $\mathrm{Mn}^{2+}$ in octahedral ligands and the ligand field strength $\Delta$ is 1.88 $\mathrm{eV}$ at $\mathrm{GGA}+U$ level and $1.87 \mathrm{eV}$ at HSE06 level. For degenerate $\mathrm{Mn}^{3+}{ }^{5} \mathrm{E}$ ground state in octahedral ligands, the average Mn-O bond length is $2.03 \AA$ (see in Fig. S8) and the ligand field strength $\Delta$ is $2.24 \mathrm{eV}$ at GGA $+U$ level and $2.30 \mathrm{eV}$ at HSE06 level. The extra 0.5e is added to ensure all five $3 d$ orbitals in the bandgap. While for the gound state geometric configuration of $\mathrm{Mn}^{4+}$ in octahedral ligands, the average Mn-O bond length is $1.94 \AA$ (see in Fig. S8). The ligand field strength $\Delta$ of $\mathrm{Mn}^{4+}$ is $2.38 \mathrm{eV}$ at GGA $+U$ level and $2.77 \mathrm{eV}$ at HSE06 level, which is estimated by the energy difference of ${ }^{4} \mathrm{~T}_{2}$ and ${ }^{4} \mathrm{~A}_{2}$. For comparison, 
the $\Delta$ for $\mathrm{Mn}^{2+}$ and $\mathrm{Mn}^{4+}$ are $1.0^{10}$ and $2.4 \mathrm{eV}^{11}$ by experiments, respectively.

The detailed ligand field strength $\Delta$ and the radio of average Mn-O bond length $R$ relative to $R_{0}=2.0 \AA$ are thus plotted in Fig. S5 and at GGA $+U$ level (a) and HSE06 level (b) and the simple model of $\Delta=A\left(R_{0}\right)\left(R_{0} / R\right)^{n}$ is applied. In Fig. S5(a) of GGA $+U$ method, the $n$ values are 2.31 and 4.62 for octahedral and tetrahedral Mn, respectively. Besides, in Fig. S5(b) of HSE06 method, the $n$ values are 3.94 and 3.67 for octahedral and tetrahedral Mn, respectively, which is close with each other and both are smaller than the parameter of point charge electrostatic model. ${ }^{12}$ The tetrahedral Mn substituted at Zn site shows smaller bond length relative to that of octahedral Mn substituted at Ga site for the same valence state.
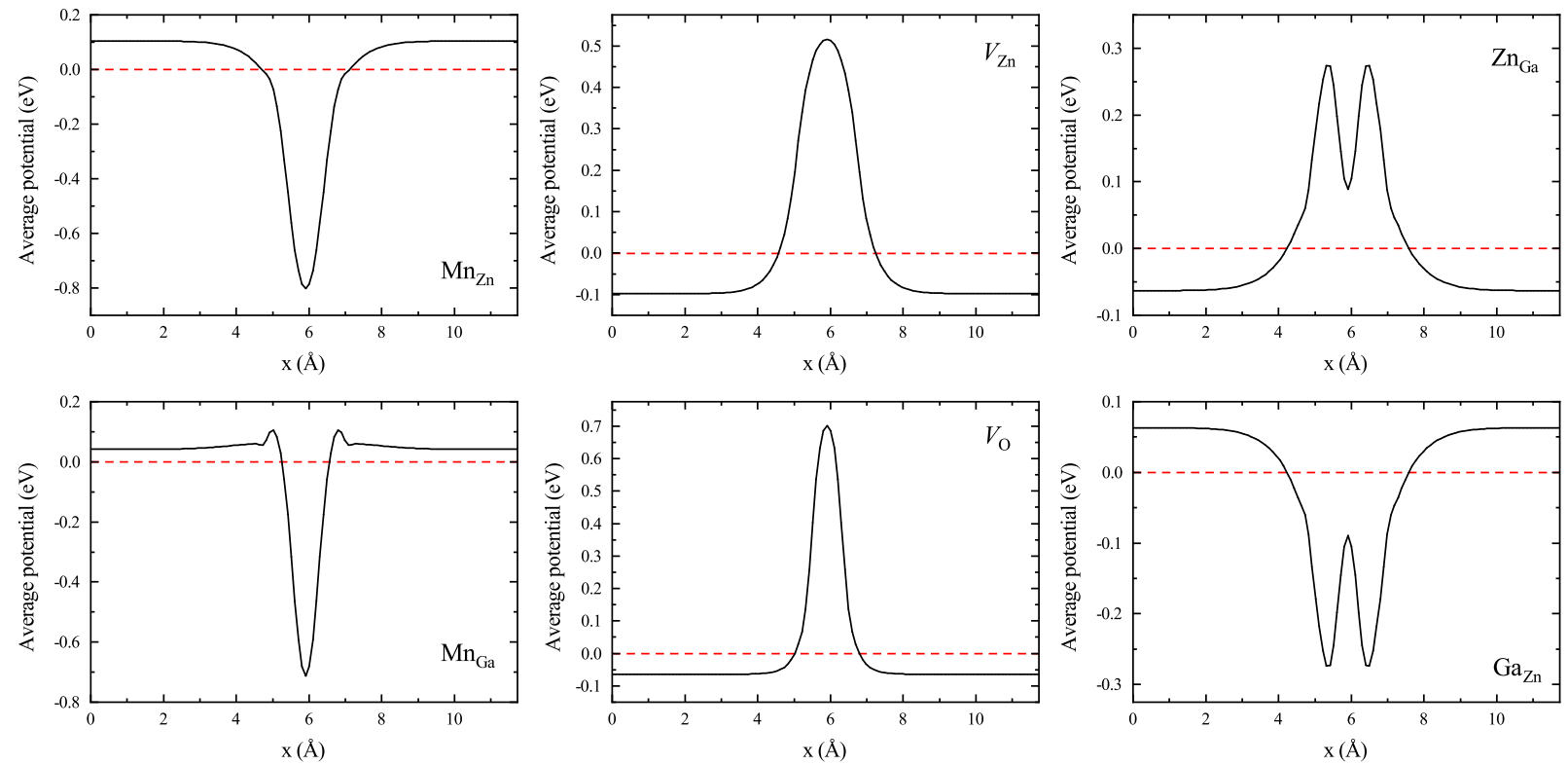

Figure S1: The potential energy of the $\mathrm{Mn}_{\mathrm{Zn}}, \mathrm{Mn}_{\mathrm{Ga}}, V_{\mathrm{Zn}}, V_{\mathrm{O}}, \mathrm{Zn}_{\mathrm{Ga}}$ and GaZn defects placed in an empty periodic cell of the same size with $\mathrm{ZnGa}_{2} \mathrm{O}_{4}$ supercell host. 
Table S1: The charge corrections to total energies of defects in $\mathrm{ZnGa}_{2} \mathrm{O}_{4}$ host

\begin{tabular}{ccccccc}
\hline \hline & $\mathrm{Mn}_{\mathrm{Zn}}$ & $\mathrm{Mn}_{\mathrm{Ga}}$ & $V_{\mathrm{Zn}}$ & $V_{\mathrm{O}}$ & $\mathrm{Zn}_{\mathrm{Ga}}$ & $\mathrm{Ga}_{\mathrm{Zn}}$ \\
\hline$\epsilon_{0}$ & \multicolumn{5}{c}{10.86} \\
$E_{\mathrm{corr}}^{\mathrm{LZ}}$ & & $0.12 q^{2}$ & & \\
$q \Delta V$ & $0.10 q$ & $0.04 q$ & $-0.10 q$ & $-0.06 q$ & $-0.06 q$ & $0.06 q$ \\
\hline \hline
\end{tabular}
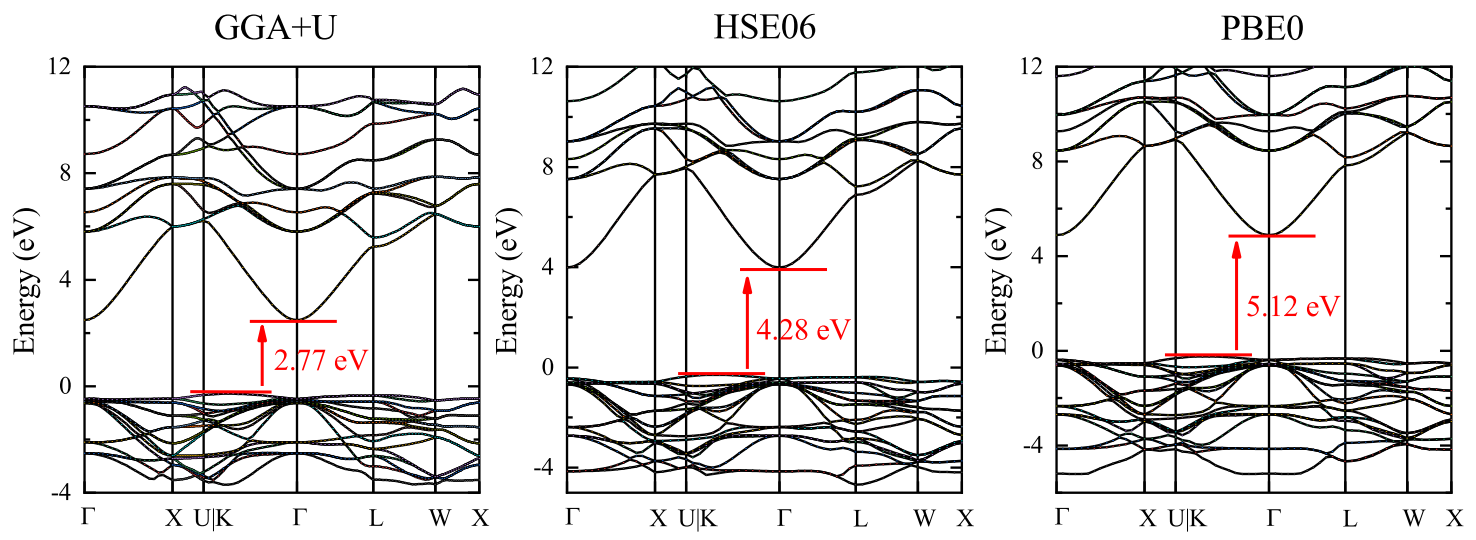

Figure S2: The band structures of pristine $\mathrm{ZnGa}_{2} \mathrm{O}_{4}$ crystal calculated by $\mathrm{GGA}+U$, HSE06 and $\operatorname{PBE} 0(\alpha)$ method.

(a) $A$ point $(-3.35,-4.99,0)$

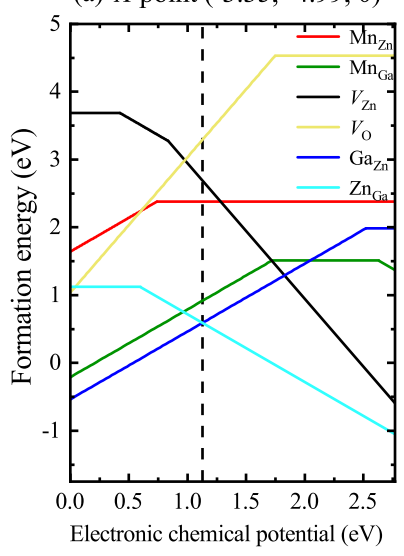

(b) $B$ point $(-3.81,-4.76,0)$

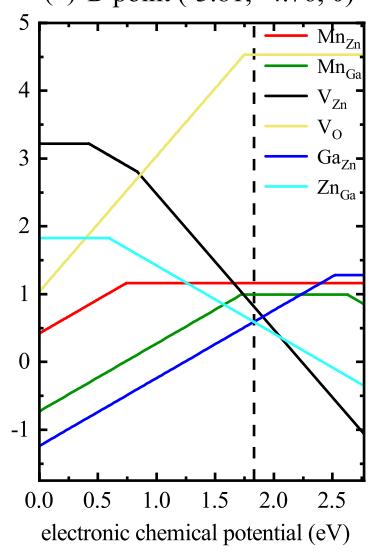

(c) $C$ point $(-0.01,0,-3.33)$

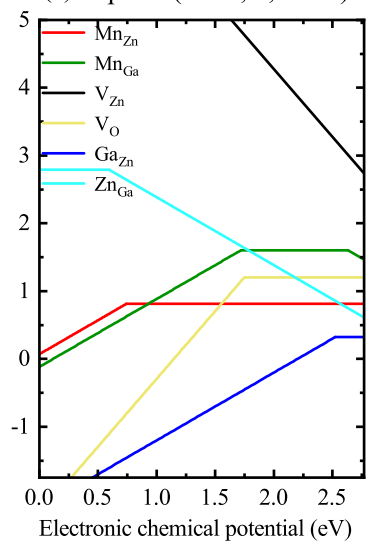

(d) $D$ point $(-0.64,0,-3.17)$

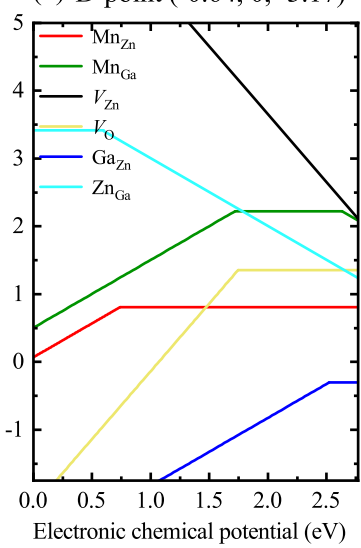

Figure S3: Formation energies of intrinsic defects and Mn impurities under determined chemical potentials of $A$ point with oxygen-rich $\Delta \mu_{\mathrm{O}}=0 \mathrm{eV}$ and rich $\mathrm{ZnO}$ (a), $B$ point with oxygen-rich $\Delta \mu_{\mathrm{O}}=0 \mathrm{eV}$ and rich $\mathrm{Ga}_{2} \mathrm{O}_{3}$ (b), $C$ point with oxygen-poor $\Delta \mu_{\mathrm{O}}=-3.33$ $\mathrm{eV}$ and rich $\mathrm{ZnO}(\mathrm{c})$ and $D$ point with oxygen-poor $\Delta \mu_{\mathrm{O}}=-3.17 \mathrm{eV}$ and rich $\mathrm{Ga}_{2} \mathrm{O}_{3}(\mathrm{~d})$ conditions. 

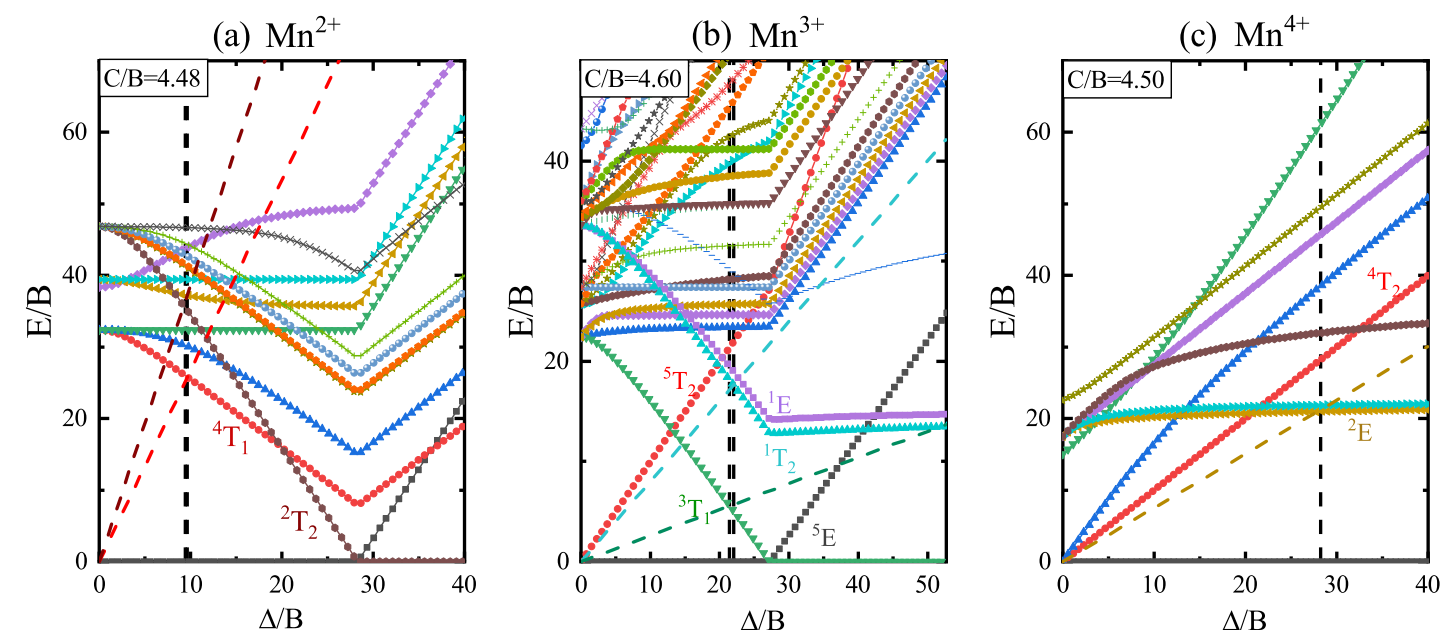

Figure S4: The Tanabe-Sugano diagram ${ }^{4}$ for $\mathrm{Mn}^{2+}$ valence state in tetrahedral ligands (a), $\mathrm{Mn}^{3+}$ (b) and $\mathrm{Mn}^{4+}$ (c) valence states in octahedral ligands, and the dashed lines are the DFT obtained parameters in corresponding ligand environments at HSE06 level.
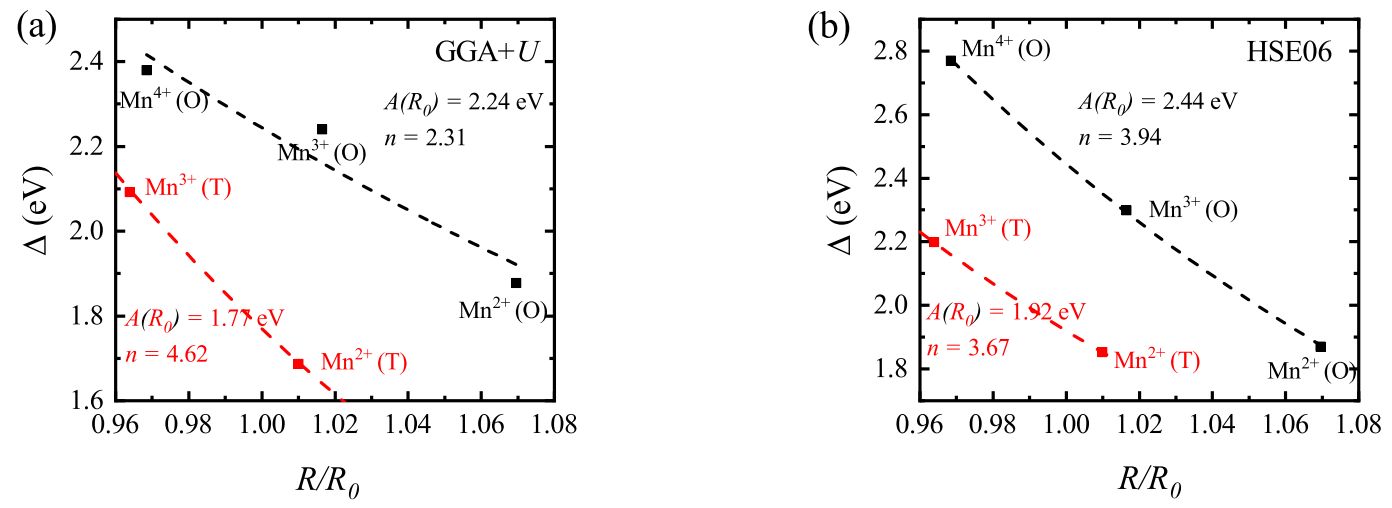

Figure S5: The ligand field strength $\Delta$ and the ratio of average $\mathrm{Mn}-\mathrm{O}$ bond length $R$ relative to $R_{0}=2.0 \AA$ of octahedral $(\mathrm{O}) \mathrm{Mn}^{2+}, \mathrm{Mn}^{3+}, \mathrm{Mn}^{4+}$ states (black) and tetrahedral (T) $\mathrm{Mn}^{2+}$ and $\mathrm{Mn}^{3+}$ states (red) at GGA $+U$ level (a) and HSE06 level (b). The dotted black and red lines are $\Delta=A\left(R_{0}\right)\left(R_{0} / R\right)^{n}$ with $A\left(R_{0}\right)$ and $n$ determined by $\Delta$ and $R$ from DFT calculations.

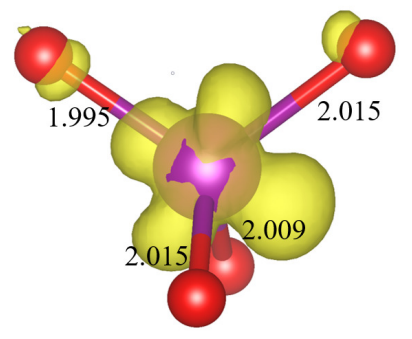

Figure S6: The partial charge density of minority-spin occupied $e$ orbital in the geometric configuration of ${ }^{4} \mathrm{~T}_{1}$ excited state. 
Table S2: The parameters obtained by DFT calculations and Tanabe-Sugano models. (T) and $(\mathrm{O})$ represent the $\mathrm{Mn}$ in tetraheral and octahedral ligands, respectively.

\begin{tabular}{ccccc}
\hline \hline Method & valence state & $\Delta(\mathrm{eV})$ & $\Delta / B$ & $B\left(\mathrm{~cm}^{-1}\right)$ \\
\hline GGA $+U$ & $\mathrm{Mn}^{2+}(\mathrm{T})$ & 0.75 & $9.3 \sim 9.7$ & $624 \sim 650$ \\
& $\mathrm{Mn}^{3+}(\mathrm{O})$ & 2.24 & $22.2 \sim 22.8$ & $792 \sim 814$ \\
& $\mathrm{Mn}^{4+}(\mathrm{O})$ & 2.38 & 27.9 & 688 \\
HSE06 & $\mathrm{Mn}^{2+}(\mathrm{T})$ & 0.82 & $9.4 \sim 9.6$ & $689 \sim 704$ \\
& $\mathrm{Mn}^{3+}(\mathrm{O})$ & 2.30 & $21.4 \sim 22.0$ & $843 \sim 867$ \\
& $\mathrm{Mn}^{4+}(\mathrm{O})$ & 2.77 & 28.2 & 792 \\
\hline \hline
\end{tabular}

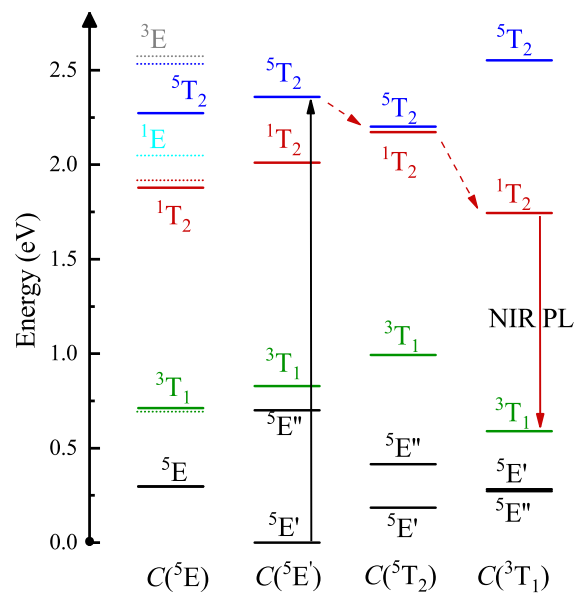

Figure S7: The energy levels calculated at the equilibrium geometric structures of ${ }^{5} \mathrm{E}$ (no JT distortion), ${ }^{5} \mathrm{E}^{\prime}$ (JT distorted), ${ }^{5} \mathrm{~T}_{2}$ and ${ }^{3} \mathrm{~T}_{1}$ states at GGA $+U$ level. The dotted energy levels are obtained with the complement of the Tanabe-Sugano model. 

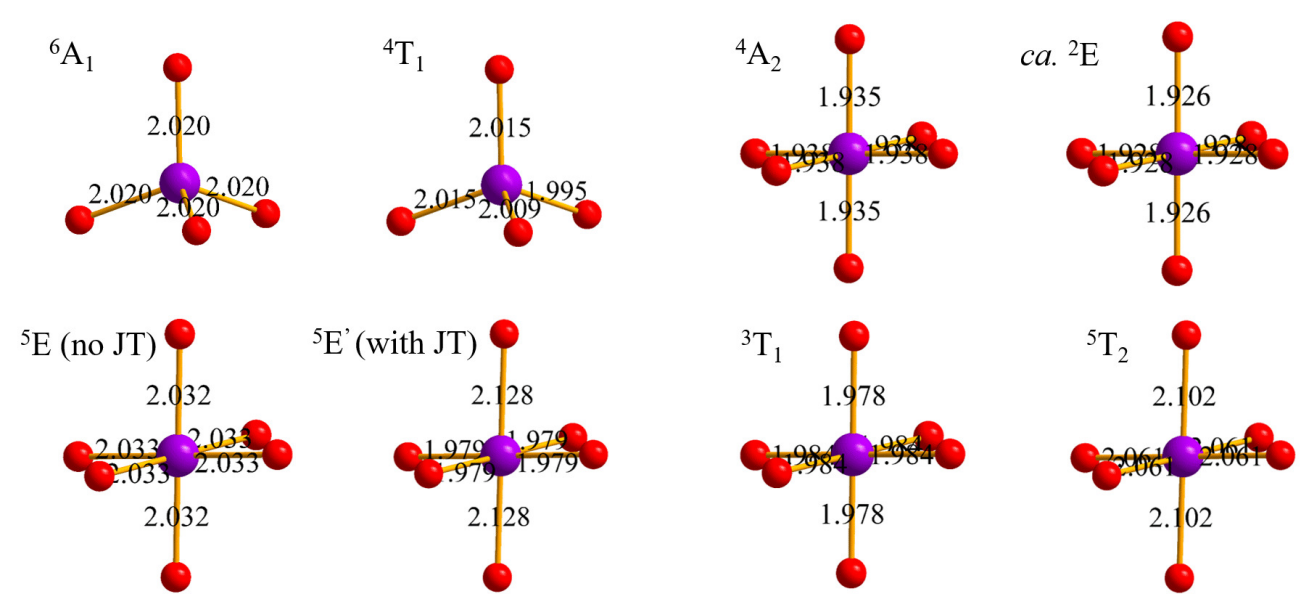

Figure S8: The equilibrium geometric configurations of $\mathrm{Mn}^{2+}{ }^{6} \mathrm{~A}_{1}$ and ${ }^{4} \mathrm{~T}_{1}$ states, $\mathrm{Mn}^{4+}{ }^{4} \mathrm{~A}_{2}$ and $c a .{ }^{2} \mathrm{E}$ (two-thirds way of ${ }^{4} \mathrm{~A}_{2}$ to ${ }^{2} \mathrm{E}$ in fact) states and $\mathrm{Mn}^{3+}{ }^{5} \mathrm{E}$ (no JT), ${ }^{5} \mathrm{E}^{\prime}$ (with JT), ${ }^{3} \mathrm{~T}_{1}$ and ${ }^{5} \mathrm{~T}_{2}$ states. Here JT stands for Jahn-Teller distortion.

\section{References}

(1) Durrant, T. R.; Murphy, S. T.; Watkins, M. B.; Shluger, A. L. Relation between image charge and potential alignment corrections for charged defects in periodic boundary conditions. The Journal of chemical physics 2018, 149, 024103.

(2) Lany, S.; Zunger, A. Assessment of correction methods for the band-gap problem and for finite-size effects in supercell defect calculations: Case studies for $\mathrm{ZnO}$ and GaAs. Physical Review B 2008, 78, 235104.

(3) Lany, S.; Zunger, A. Accurate prediction of defect properties in density functional supercell calculations. Modelling and simulation in materials science and engineering 2009, 17, 084002.

(4) Sugano, S. Multiplets of transition-metal ions in crystals; Elsevier, 2012.

(5) Gilardoni, F.; Weber, J.; Bellafrouh, K.; Daul, C.; Güdel, H. U. Excited state properties of $\mathrm{Cr}^{3+}$ in $\mathrm{Cs}_{2} \mathrm{NaYCl}_{6}$ and $\mathrm{Cs}_{2} \mathrm{NaYBr}_{6}$ : A density functional study. The Journal of chemical physics 1996, 104, 7624-7632.

(6) Lou, B.; Wen, J.; Cai, J.; Yeung, Y.-Y.; Yin, M.; Duan, C.-K. First-principles study 
of $\mathrm{Bi}^{3+}$-related luminescence and traps in the perovskites $\mathrm{CaMO}_{3}(\mathrm{M}=\mathrm{Zr}, \mathrm{Sn}, \mathrm{Ti})$. Physical Review B 2021, 103, 075109.

(7) Jia, Y.; Poncé, S.; Miglio, A.; Mikami, M.; Gonze, X. Assessment of first-principles and semiempirical methodologies for absorption and emission energies of $\mathrm{Ce}^{3+}$-doped luminescent materials. Advanced Optical Materials 2017, 5, 1600997.

(8) Joos, J. J.; Van der Heggen, D.; Martin, L. I.; Amidani, L.; Smet, P. F.; Barandiarán, Z.; Seijo, L. Broadband infrared LEDs based on europium-to-terbium charge transfer luminescence. Nature communications 2020, 11, 1-11.

(9) Henderson, B.; Imbusch, G. F. Optical spectroscopy of inorganic solids; Oxford University Press, 2006; Vol. 44.

(10) Lv, S.; Shanmugavelu, B.; Wang, Y.; Mao, Q.; Zhao, Y.; Yu, Y.; Hao, J.; Zhang, Q.; Qiu, J.; Zhou, S. Transition metal doped smart glass with pressure and temperature sensitive luminescence. Advanced Optical Materials 2018, 6, 1800881.

(11) Adachi, S. $\mathrm{Mn}^{4+}$-activated red and deep red-emitting phosphors. ECS Journal of Solid State Science and Technology 2019, 9, 016001.

(12) Tanner, P. A.; Ning, L. Electronegativity, charge transfer, crystal field strength, and the point charge model revisited. The Journal of Physical Chemistry A 2013, 117, 1503-1507. 